\title{
Oscillatory Behavior of a Class of Second-order Nonlinear Dynamic Equations on Time Scales
}

\author{
Da-Xue Chen, Guang-Hui Liu
}

College of Science Hunan Institute of Engineering 88 East Fuxing Road, Xiangtan 411104, Hunan, P. R. China

\begin{abstract}
The paper is devoted to the oscillation of a class of second-order nonlinear dynamic equations on time scales. By developing a generalized Riccati transformation technique, we establish some oscillation criteria for all solutions of the equations. Our results improve and extend some known results in the literature.
\end{abstract}

Index Terms: Oscillation, second-order nonlinear dynamic equation, time scale.

(C) 2011 Published by MECS Publisher. Selection and/or peer review under responsibility of the Research Association of Modern Education and Computer Science.

\section{Introduction}

The theory of dynamic equations on time scales is a fairly new theory. Not only can the theory unify the theories of differential equations and difference equations, but it is also able to extend these classical cases to cases "in between," e.g., to the so-called $q$-difference equations. The general idea is to prove a result for a dynamic equation where the domain of the unknown function is a time scale $T$. In this way results not only related to the set of real numbers $\mathrm{R}$ or the set of integers $\mathrm{Z}$ but those pertaining to more general time scales are obtained. There are many applications of dynamic equations on time scales to biology, quantum mechanics, electrical engineering, neural networks, heat transfer, combinatorics, social sciences and so on. A time scale $\mathrm{T}$ is an arbitrary nonempty closed subset of the real numbers $\mathrm{R}$. A book on the subject of time scales, by Bohner and Peterson [1], summarizes and organizes much of time scale calculus, see also the book by Bohner and Peterson [2] for advances in dynamic equations on time scales.

In this paper, we deal with the oscillation problem for the following second-order nonlinear dynamic equation on an arbitrary time scale T, where the following conditions are assumed to hold:

$$
\left(r(t)\left|x^{\Delta}(t)\right|^{\alpha-1} x^{\Delta}(t)\right)^{\Delta}+q(t)|x(t)|^{\beta-1} x(t)=0
$$

* Corresponding author.

E-mail address: cdx2003@163.com,1gh233@163.com 
$\left(\mathrm{S}_{1}\right) \alpha, \beta>0$ are constants and sup $\mathrm{T}=\infty$;

$\left(\mathrm{S}_{2}\right) r$ and $q$ are positive rd-continuous functions defined on the time scale interval $\left[t_{0}, \infty\right)$.

By a solution of (1.1) we mean a nontrivial real-valued function $x \in C_{r d}^{1}\left[t_{x}, \infty\right)$ for a certain $t_{x} \geq t_{0}$, which has the property that $r(t)\left|x^{\Delta}(t)\right|^{\alpha-1} x^{\Delta}(t) \in C_{r d}^{1}\left[t_{x}, \infty\right)$ and satisfies (1.1) for $t \geq t_{x}$. Our attention is restricted to those solutions of (1.1) which exist on the half-line $\left[t_{x}, \infty\right)$ and satisfy $\sup \left\{|x(t)|: t>t_{*}\right\}>0$ for any $t_{*} \geq t_{x}$. A solution $x$ of (1.1) is said to be oscillatory if it is neither eventually positive nor eventually negative, otherwise it is nonoscillatory. Equation (1.1) is said to be oscillatory if all its solutions are oscillatory.

During the past years, the oscillation theory of dynamic equations has been developed very rapidly. For some papers on the subject, we refer to [3-8] and the references cited therein.

In 2005, Saker [4] presented some oscillation criteria for (1.1) when $\alpha=\beta>1$ is an odd positive integer and $\left(\mathrm{S}_{2}\right)$ holds. In 2008, Hassan [5] obtained some sufficient conditions for the oscillation of (1.1) when $\alpha=\beta$ is a quotient of odd positive integers and $\left(\mathrm{S}_{2}\right)$ holds. Hassan [5] improved and extended the results of Saker [4]. In the last year, Grace et al. [6] established several new oscillation criteria for (1.1) when $\alpha, \beta$ are quotients of odd positive integers and $\left(\mathrm{S}_{2}\right)$ holds.

However, the cases considered by [4-6] are some special cases of (1.1), and all the results of [4-6] can not be applied to (1.1) when $\alpha, \beta$ are not equal to quotients of odd positive integers. Thus, it is of great interest to investigate the oscillation of (1.1) when $\alpha, \beta>0$ are constants. In this paper, we will establish some new oscillation criteria for (1.1) when $\alpha, \beta>0$ are constants. Our results improve and extend the results of [4-6].

The following lemmas are useful in the proof of our main results.

Lemma 1.1. (Bohner and Peterson [1], p. 32, Theorem 1.87) Let $f: \mathrm{R} \rightarrow \mathrm{R}$ be continuously differentiable and suppose $g: \mathrm{T} \rightarrow \mathrm{R}$ is delta differentiable. Then $f \circ g: \mathrm{T} \rightarrow \mathrm{R}$ is delta differentiable and satisfies

$$
(f \circ g)^{\Delta}(t)=\left\{\int_{0}^{1} f^{\prime}\left(g(t)+h \mu(t) g^{\Delta}(t)\right) d h\right\} g^{\Delta}(t),
$$

where $\mu(t):=\sigma(t)-t$ is the graininess function on $\mathrm{T}$, here $\sigma(t):=\inf \{s \in \mathrm{Tt} s>t\}$ is the forward jump operator on $\mathrm{T}$.

Lemma 1.2. (Hardy et al. [9]) If $X$ and $Y$ are nonnegative, then

$$
\lambda X Y^{\lambda-1}-X^{\lambda} \leq(\lambda-1) Y^{\lambda} \text { when } \lambda>1,
$$

where the equality holds if and only if $X=Y$.

\section{Main Results}

Theorem 2.1. Assume that $\left(\mathrm{S}_{1}\right),\left(\mathrm{S}_{2}\right)$ and the following condition hold:

$$
\int_{t_{0}}^{\infty} r^{-1 / \alpha}(t) \Delta t=\infty \text {. }
$$

Furthermore, assume that there exists a positive nondecreasing delta differentiable function $\varphi$ such that for all $T>t_{1} \geq t_{0}$, 
$\limsup _{t \rightarrow \infty} \int_{T}^{t}\left[q(s) \varphi(s)-\frac{(\alpha / \beta)^{\alpha} r(s)\left(\varphi^{\Delta}(s)\right)^{\alpha+1}}{(\alpha+1)^{\alpha+1}(\varphi(s) v(s))^{\alpha}}\right] \Delta s=\infty$

where $v(t):= \begin{cases}c_{1}, & \text { if } \alpha<\beta, \\ 1, & \text { if } \alpha=\beta, \\ c_{2}\left(u^{\sigma}(t)\right)^{(\alpha-\beta) / \alpha}, & \text { if } \alpha>\beta,\end{cases}$

here $c_{1}$ and $c_{2}$ are any positive constants, $\sigma$ is the forward jump operator on $\mathrm{T}$, $u(t):=\left(\int_{t_{1}}^{t} r^{-1 / \alpha}(s) \Delta s\right)^{-1}$ and $u^{\sigma}:=u \circ \sigma$. Then (1.1) is oscillatory.

Proof. Let $x$ be a nonoscillatory solution of (1.1). Without loss of generality, we may assume that $x$ is an eventually positive solution of (1.1). Then there exists $t_{1} \geq t_{0}$ such that $x(t)>0$ for $t \in\left[t_{1}, \infty\right)$. Therefore, from (1.1) we have

$$
\left(r(t)\left|x^{\Delta}(t)\right|^{\alpha-1} x^{\Delta}(t)\right)^{\Delta}=-q(t) x^{\beta}(t)<0
$$

for $t \geq t_{1}$. Thus, we see that $r(t)\left|x^{\Delta}(t)\right|^{\alpha-1} x^{\Delta}(t)$ is strictly decreasing on $\left[t_{1}, \infty\right)$ and is eventually of one sign. We claim $x^{\Delta}(t)>0$ for $t \in\left[t_{1}, \infty\right)$. Assume on the contrary, then there exists $t_{2} \geq t_{1}$ such that $x^{\Delta}\left(t_{2}\right) \leq 0$. Take $t_{3}>t_{2}$. Then we obtain

$$
\begin{aligned}
r(t)\left|x^{\Delta}(t)\right|^{\alpha-1} x^{\Delta}(t) & \leq r\left(t_{3}\right)\left|x^{\Delta}\left(t_{3}\right)\right|^{\alpha-1} x^{\Delta}\left(t_{3}\right) \\
& <r\left(t_{2}\right)\left|x^{\Delta}\left(t_{2}\right)\right|^{\alpha-1} x^{\Delta}\left(t_{2}\right)
\end{aligned}
$$

for $t \in\left[t_{3}, \infty\right)$. Let $M:=r\left(t_{3}\right)\left|x^{\Delta}\left(t_{3}\right)\right|^{\alpha-1} x^{\Delta}\left(t_{3}\right)<0$. Then we get $x^{\Delta}(t) \leq-(-M)^{1 / \alpha} r^{-1 / \alpha}(t)$ for $t \in\left[t_{3}, \infty\right)$. Integrating both sides of the last inequality from $t_{3}$ to $t$, we have

$$
x(t)-x\left(t_{3}\right) \leq-(-M)^{1 / \alpha} \int_{t_{3}}^{t} r^{-1 / \alpha}(s) \Delta s
$$

for $t \in\left[t_{3}, \infty\right)$. Letting $t \rightarrow \infty$ and using (2.1), we conclude $\lim _{t \rightarrow \infty} x(t)=-\infty$. This contradicts the fact that $x(t)>0$ for $t \in\left[t_{1}, \infty\right)$. Thus, we have $x^{\Delta}(t)>0$ for $t \in\left[t_{1}, \infty\right)$. Let $w(t):=r(t)\left(x^{\Delta}(t)\right)^{\alpha} \frac{\varphi(t)}{x^{\beta}(t)}$ for $t \in\left[t_{1}, \infty\right)$. Then by the formulas

$$
(F G)^{\Delta}=F^{\Delta} G+F^{\sigma} G^{\Delta}
$$

and

$$
(F / G)^{\Delta}=F^{\Delta} / G^{\sigma}-F G^{\Delta} /\left(G G^{\sigma}\right)
$$

for the delta derivatives of the product $F G$ and the quotient $F / G$ of differentiable functions $F$ and $G$, where $\sigma$ is the forward jump operator on $\mathrm{T}, F^{\sigma}:=F \circ \sigma$ and $G^{\sigma}:=G \circ \sigma$, we get 


$$
\begin{aligned}
w^{\Delta} & =\left(r\left(x^{\Delta}\right)^{\alpha}\right)^{\Delta} \frac{\varphi}{x^{\beta}}+\left(r\left(x^{\Delta}\right)^{\alpha}\right)^{\sigma}\left(\frac{\varphi}{x^{\beta}}\right)^{\Delta} \\
& =\left(r\left(x^{\Delta}\right)^{\alpha}\right)^{\Delta} \frac{\varphi}{x^{\beta}}+\left(r\left(x^{\Delta}\right)^{\alpha}\right)^{\sigma}\left(\frac{\varphi^{\Delta}}{\left(x^{\beta}\right)^{\sigma}}-\frac{\varphi\left(x^{\beta}\right)^{\Delta}}{x^{\beta}\left(x^{\beta}\right)^{\sigma}}\right) .
\end{aligned}
$$

For $t \geq t_{1}$, since $\left(r(t)\left(x^{\Delta}(t)\right)^{\alpha}\right)^{\Delta}=-q(t) x^{\beta}(t)$, we have

$$
\begin{aligned}
w^{\Delta} & =-q \varphi+\left(r\left(x^{\Delta}\right)^{\alpha}\right)^{\sigma}\left(\frac{\varphi^{\Delta}}{\left(x^{\beta}\right)^{\sigma}}-\frac{\varphi\left(x^{\beta}\right)^{\Delta}}{x^{\beta}\left(x^{\beta}\right)^{\sigma}}\right) \\
& =-q \varphi+\frac{w^{\sigma}}{\varphi^{\sigma}} \varphi^{\Delta}-\varphi \frac{w^{\sigma}}{\varphi^{\sigma}} \frac{\left(x^{\beta}\right)^{\Delta}}{x^{\beta}} .
\end{aligned}
$$

For $t \in\left[t_{1}, \infty\right)$, since $0<x(t) \leq x^{\sigma}(t)$, by Lemma 1.1 and by the formula $x^{\sigma}(t)=x(t)+\mu(t) x^{\Delta}(t)$ we obtain

$$
\begin{aligned}
\left(x^{\beta}(t)\right)^{\Delta} & =\beta x^{\Delta}(t) \int_{0}^{1}\left[x(t)+h \mu(t) x^{\Delta}(t)\right]^{\beta-1} d h \\
& =\beta x^{\Delta}(t) \int_{0}^{1}\left[(1-h) x(t)+h x^{\sigma}(t)\right]^{\beta-1} d h \\
& \geq \begin{cases}\beta x^{\Delta}(t) \int_{0}^{1}\left(x^{\sigma}(t)\right)^{\beta-1} d h, & \text { if } 0<\beta \leq 1, \\
\beta x^{\Delta}(t) \int_{0}^{1} x^{\beta-1}(t) d h, & \text { if } \beta>1\end{cases} \\
& = \begin{cases}\beta\left(x^{\sigma}(t)\right)^{\beta-1} x^{\Delta}(t), & \text { if } 0<\beta \leq 1, \\
\beta x^{\beta-1}(t) x^{\Delta}(t), & \text { if } \beta>1 .\end{cases}
\end{aligned}
$$

Therefore, for $t \in\left[t_{1}, \infty\right)$ we conclude

$$
\frac{\left(x^{\beta}(t)\right)^{\Delta}}{x^{\beta}(t)} \geq \begin{cases}\beta\left(x^{\sigma}(t)\right)^{\beta-1} x^{\Delta}(t) / x^{\beta}(t), & \text { if } 0<\beta \leq 1, \\ \beta x^{\Delta}(t) / x(t), & \text { if } \beta>1 .\end{cases}
$$

For $t \in\left[t_{1}, \infty\right)$, since $0<x(t) \leq x^{\sigma}(t)$, we obtain

$$
\left(x^{\beta}(t)\right)^{\Delta} / x^{\beta}(t) \geq \beta x^{\Delta}(t) / x^{\sigma}(t) \text { for all } \beta>0 .
$$

Hence, from (2.3) we find

$$
w^{\Delta} \leq-q \varphi+\frac{w^{\sigma}}{\varphi^{\sigma}} \varphi^{\Delta}-\beta \varphi \frac{w^{\sigma}}{\varphi^{\sigma}} \frac{x^{\Delta}}{x^{\sigma}} \quad \text { on }\left[t_{1}, \infty\right) .
$$

From the definition of $w$ we get $r^{1 / \alpha} x^{\Delta}=\left(w x^{\beta} / \varphi\right)^{1 / \alpha}$.

Since $r^{1 / \alpha}(t) x^{\Delta}(t)$ is strictly decreasing and $t \leq \sigma(t)$ on $\left[t_{1}, \infty\right)$, we have 


$$
r^{1 / \alpha} x^{\Delta} \geq\left(r^{1 / \alpha} x^{\Delta}\right)^{\sigma}=\left(w^{\sigma}\left(x^{\sigma}\right)^{\beta} / \varphi^{\sigma}\right)^{1 / \alpha} \text { on }\left[t_{1}, \infty\right) .
$$

Thus, from (2.4) we obtain for $t \in\left[t_{1}, \infty\right)$

$$
w^{\Delta} \leq-q \varphi+\frac{w^{\sigma}}{\varphi^{\sigma}} \varphi^{\Delta}-\frac{\beta \varphi\left(w^{\sigma}\right)^{(1+\alpha) / \alpha}}{r^{1 / \alpha}\left(\varphi^{\sigma}\right)^{(1+\alpha) / \alpha}}\left(x^{\sigma}\right)^{(\beta-\alpha) / \alpha} .
$$

Next, we consider the following three cases:

Case (i). Let $\alpha<\beta$. For $t \in\left[t_{1}, \infty\right)$, since $x^{\sigma}(t) \geq x(t) \geq x\left(t_{1}\right)>0$, we have

$$
\left(x^{\sigma}\right)^{(\beta-\alpha) / \alpha}(t) \geq\left(x\left(t_{1}\right)\right)^{(\beta-\alpha) / \alpha}:=c_{1} .
$$

Case (ii). Let $\alpha=\beta$. Then, for $t \in\left[t_{1}, \infty\right)$ we get

$\left(x^{\sigma}\right)^{(\beta-\alpha) / \alpha}(t)=1$.

Case (iii). Let $\alpha>\beta$. Since $r(t)\left(x^{\Delta}(t)\right)^{\alpha}$ is strictly decreasing on $\left[t_{1}, \infty\right)$, for $t \in\left[t_{1}, \infty\right)$ we have

$$
r(t)\left(x^{\Delta}(t)\right)^{\alpha} \leq r\left(t_{1}\right)\left(x^{\Delta}\left(t_{1}\right)\right)^{\alpha}:=b .
$$

Hence, we obtain $x^{\Delta}(t) \leq b^{1 / \alpha} r^{-1 / \alpha}(t)$ for $t \in\left[t_{1}, \infty\right)$. Integrating both sides of the last inequality from $t_{1}$ to $t$, we have

$$
x(t) \leq x\left(t_{1}\right)+b^{1 / \alpha} \int_{t_{1}}^{t} r^{-1 / \alpha}(s) \Delta s \text { for } t \in\left[t_{1}, \infty\right) .
$$

Therefore, there exist a constant $b_{1}>0$ and $t_{4}>t_{1}$ such that $x(t) \leq b_{1} \int_{t_{1}}^{t} r^{-1 / \alpha}(s) \Delta s:=b_{1} u^{-1}(t)$ for $t \in\left[t_{4}, \infty\right)$. Hence, for $t \in\left[t_{4}, \infty\right)$ we get

$$
\left(x^{\sigma}(t)\right)^{(\beta-\alpha) / \alpha} \geq c_{2}\left(u^{\sigma}(t)\right)^{(\alpha-\beta) / \alpha},
$$

where $c_{2}:=\left(b_{1}\right)^{(\beta-\alpha) / \alpha}$.

Thus, for all $\alpha, \beta>0$ and for $t \in\left[t_{4}, \infty\right)$, from (2.5)-(2.8) it follows that

$$
w^{\Delta} \leq-q \varphi+\frac{w^{\sigma}}{\varphi^{\sigma}} \varphi^{\Delta}-\frac{\beta \varphi\left(w^{\sigma}\right)^{(1+\alpha) / \alpha}}{r^{1 / \alpha}\left(\varphi^{\sigma}\right)^{(1+\alpha) / \alpha}} v .
$$

Taking $\lambda=(\alpha+1) / \alpha, X=\frac{(\beta \varphi v)^{1 / \lambda}}{r^{1 /(\alpha+1)} \varphi^{\sigma}} w^{\sigma}$ and $Y=\frac{\left(\varphi^{\Delta}\right)^{\alpha} r^{1 / \lambda}}{\lambda^{\alpha}(\beta \varphi v)^{\alpha / \lambda}}$, by (2.9) and Lemma 1.2 we obtain

$$
w^{\Delta} \leq-q \varphi+\frac{(\alpha / \beta)^{\alpha} r\left(\varphi^{\Delta}\right)^{\alpha+1}}{(\alpha+1)^{\alpha+1}(\varphi v)^{\alpha}} \text { on }\left[t_{4}, \infty\right)
$$


Integrating both sides of the last inequality from $t_{4}$ to $t$, we obtain for $t \in\left[t_{4}, \infty\right)$

$$
\begin{aligned}
& \int_{t_{4}}^{t}\left[q(s) \varphi(s)-\frac{(\alpha / \beta)^{\alpha} r(s)\left(\varphi^{\Delta}(s)\right)^{\alpha+1}}{(\alpha+1)^{\alpha+1}(\varphi(s) v(s))^{\alpha}}\right] \Delta s \\
& \leq w\left(t_{4}\right)-w(t)<w\left(t_{4}\right) .
\end{aligned}
$$

Hence, we get

$$
\begin{aligned}
& \limsup _{t \rightarrow \infty} \int_{t_{4}}^{t}\left[q(s) \varphi(s)-\frac{(\alpha / \beta)^{\alpha} r(s)\left(\varphi^{\Delta}(s)\right)^{\alpha+1}}{(\alpha+1)^{\alpha+1}(\varphi(s) v(s))^{\alpha}}\right] \Delta s \\
& \leq w\left(t_{4}\right)<\infty,
\end{aligned}
$$

which contradicts (2.2). The proof is complete.

Next, we consider the case when

$$
\int_{t_{0}}^{\infty} r^{-1 / \alpha}(t) \Delta t<\infty
$$

holds, which implies that (2.1) doesn't hold.

Theorem 2.2. Assume that $\left(S_{1}\right),\left(S_{2}\right)$ and (2.10) hold. Furthermore, assume that there exists a positive nondecreasing delta differentiable function $\varphi$ such that for all $T>t_{1} \geq t_{0}$, (2.2) and the following condition hold:

$$
\int_{T}^{\infty}\left(\frac{1}{r(z)} \int_{T}^{z} k^{\beta}(s) q(s) \Delta s\right)^{1 / \alpha} \Delta z=\infty,
$$

where $k(t):=\int_{t}^{\infty} r^{-1 / \alpha}(s) \Delta s$. Then (1.1) is oscillatory.

Proof. Assume that $x$ is a nonoscillatory solution of (1.1). Without loss of generality, we may assume that $x$ is an eventually positive solution of (1.1). Then there exists $t_{1} \geq t_{0}$ such that $x(t)>0$ for $t \in\left[t_{1}, \infty\right)$. Proceeding as in the proof of Theorem 2.1, we obtain that $r(t)\left|x^{\Delta}(t)\right|^{\alpha-1} x^{\Delta}(t)$ is strictly decreasing on $\left[t_{1}, \infty\right)$ and is eventually of one sign. Therefore, there are two cases for the sign of $x^{\Delta}(t)$. The proof when $x^{\Delta}(t)$ is eventually positive is similar to that of Theorem 2.1 and hence is omitted.

Next, assume that $x^{\Delta}(t)$ is eventually negative. Then there exists $t_{2} \geq t_{1}$ such that $x^{\Delta}(t)<0$ for $t \in\left[t_{2}, \infty\right)$. Thus, from (1.1) we have $\left(r(t)\left(-x^{\Delta}(t)\right)^{\alpha}\right)^{\Delta}=q(t) x^{\beta}(t)>0$ for $t \in\left[t_{2}, \infty\right)$, which implies that $r(t)\left(-x^{\Delta}(t)\right)^{\alpha}$ is strictly increasing on $\left[t_{2}, \infty\right)$. Hence, we have

$$
r(s)\left(-x^{\Delta}(s)\right)^{\alpha} \geq r(t)\left(-x^{\Delta}(t)\right)^{\alpha} \text { for } s \geq t \geq t_{2} .
$$

Then for $s \geq t \geq t_{2}$ we conclude

$$
-x^{\Delta}(s) \geq r^{-1 / \alpha}(s) r^{1 / \alpha}(t)\left(-x^{\Delta}(t)\right) .
$$

Integrating both sides of the last inequality from $t \geq t_{2}$ to $z \geq t$ and letting $z \rightarrow \infty$, for $t \in\left[t_{2}, \infty\right)$ we get 


$$
\begin{aligned}
x(t) & \geq\left(\int_{t}^{\infty} r^{-1 / \alpha}(s) \Delta s\right) r^{1 / \alpha}(t)\left(-x^{\Delta}(t)\right) \\
& :=k(t) r^{1 / \alpha}(t)\left(-x^{\Delta}(t)\right) \\
& \geq k(t) r^{1 / \alpha}\left(t_{2}\right)\left(-x^{\Delta}\left(t_{2}\right)\right) \\
& :=c k(t),
\end{aligned}
$$

where $c:=-r^{1 / \alpha}\left(t_{2}\right) x^{\Delta}\left(t_{2}\right)>0$. Thus, from (1.1) we obtain

$$
\left(r(t)\left(-x^{\Delta}(t)\right)^{\alpha}\right)^{\Delta}=q(t) x^{\beta}(t) \geq c^{\beta} k^{\beta}(t) q(t)
$$

for $t \in\left[t_{2}, \infty\right)$. Integrating both sides of the last inequality from $t_{2}$ to $t$, for $t \in\left[t_{2}, \infty\right)$ we have

$$
\begin{aligned}
r(t)\left(-x^{\Delta}(t)\right)^{\alpha} & \geq r\left(t_{2}\right)\left(-x^{\Delta}\left(t_{2}\right)\right)^{\alpha}+c^{\beta} \int_{t_{2}}^{t} k^{\beta}(s) q(s) \Delta s \\
& >c^{\beta} \int_{t_{2}}^{t} k^{\beta}(s) q(s) \Delta s .
\end{aligned}
$$

Hence, we obtain $-x^{\Delta}(t)>\left(\frac{1}{r(t)} c^{\beta} \int_{t_{2}}^{t} k^{\beta}(s) q(s) \Delta s\right)^{1 / \alpha}$ for $t \in\left[t_{2}, \infty\right)$. Integrating both sides of the last inequality from $t_{2}$ to $t$, we find

$$
x(t) \leq x\left(t_{2}\right)-c^{\beta / \alpha} \int_{t_{2}}^{t}\left(\frac{1}{r(z)} \int_{t_{2}}^{z} k^{\beta}(s) q(s) \Delta s\right)^{1 / \alpha} \Delta z
$$

for $t \in\left[t_{2}, \infty\right)$. Letting $t \rightarrow \infty$ and using (2.11), we see $\lim _{t \rightarrow \infty} x(t)=-\infty$. This contradicts the fact that $x(t)>0$ for $t \in\left[t_{1}, \infty\right)$. The proof is complete.

Remark 2.1. From Theorems 2.1 and 2.2, we can obtain many different sufficient conditions for the oscillation of (1.1) with different choices of the function $\varphi$.

For instance, let $\varphi(t)=1$, then $\varphi^{\Delta}(t)=0$ and Theorems 2.1 and 2.2 imply the following results, respectively.

Corollary 2.1. Suppose that $\left(\mathrm{S}_{1}\right),\left(\mathrm{S}_{2}\right)$ and (2.1) hold. Furthermore, assume that $\int_{t_{0}}^{\infty} q(t) \Delta t=\infty$. Then (1.1) is oscillatory.

Corollary 2.2. Suppose that $\left(\mathrm{S}_{1}\right),\left(\mathrm{S}_{2}\right),(2.10)$ and $(2.11)$ hold. Furthermore, assume that $\int_{t_{0}}^{\infty} q(t) \Delta t=\infty$. Then (1.1) is oscillatory.

\section{References}

[1] M. Bohner and A. Peterson, Dynamic Equations on Time Scales: An Introduction with Applications, Boston: Birkhäuser, 2001.

[2] M. Bohner and A. Peterson, Advances in Dynamic Equations on Time Scales, Boston: Birkhäuser, 2003.

[3] D.-X. Chen, "Oscillation and asymptotic behavior for $n$ th-order nonlinear neutral delay dynamic equations on time scales," Acta Appl. Math., vol. 109, no. 3, pp. 703-719, March 2010. 
[4] S.H. Saker, "Oscillation criteria of second-order half-linear dynamic equations on time scales," J. Comput. Appl. Math., vol. 177, no. 2, pp. 375-387, May 2005.

[5] T.S. Hassan, "Oscillation criteria for half-linear dynamic equations on time scales," J. Math. Anal. Appl., vol. 345, no. 1, pp. 176-185, September 2008.

[6] S.R. Grace, R.P. Agarwal, B. Kaymakçalan and W. Sae-Jie, "On the oscillation of certain second order nonlinear dynamic equations," Math. Comput. Modelling., vol. 50, no. 1-2, pp. 273-286, July 2009.

[7] Q. Zhang, "Oscillation of second-order half-linear delay dynamic equations with damping on time scales," J. Comput. Appl. Math., vol. 235, no. 5, pp. 1180-1188, January 2011.

[8] M. Huang, "Oscillation criteria for second order nonlinear dynamic equations with impulses," Comput. Math. Appl., vol. 59, no. 1, pp. 31-41, January 2010.

[9] G.H. Hardy, J.E. Littlewood and G. Pólya, Inequalities (second ed.), Cambridge: Cambridge University Press, 1988. 